Original Article

\title{
Extremely dilution of Strychnos Nux vomica mitigates alcohol-induced reduction in enthalpies associated with free water molecules in fish brain
}

\author{
Atheni Konar ${ }^{12^{*}}$, Tandra Sarkar ${ }^{1,2}$, Nirmal Chandra Sukul ${ }^{1,3}$, Anirban Sukul ${ }^{2}$, Amir \\ Sohel $^{4}$, Asmita Sengupta ${ }^{4}$ \\ 1 Centre for Healthcare Science and Technology, IIEST, Shibpur-711103, West Bengal, India.; 2 Sukul Institute of Homeopathic Research, Shyambati, Santiniketan-731235, West \\ Bengal, India.; 3 Department of Zoology, Visva-Bharati University, Santiniketan 731235, West Bengal, India.; 4 Department of Physics, Visva-Bharati University, Santiniketan \\ 731235, West Bengal, India. E-mail: *ak8971@gmail.com
}

\begin{abstract}
Background: Alcoholism is a global health problem. Extract of the seeds of Strychnos Nux vomica and its high dilution have long been used in homeopathy for alcohol induced diseases of patients. Alcoholism leads to reduced brain volume. Glial cells like astroglia contain large number of water channel proteins or aquaporin (AQP4) which mediate glial oedema resulting from ethanol intoxication. Nux vomica, a homeopathic drug of plant origin, is known to counteract alcohol effect.

Aim: The objective of this present study is to find out the leve

of a teleost fish under ethanol intoxication. The other purp f free water molecules in the brain could restore the level of free water in the alcohol treated fi: to determine whether Nux vomica

Methodology: One group of fish was exposed to $456 \mathrm{mM}$ ethan ol for $30 \mathrm{~min}$, another exposed first to a solution of Nux vomica 200c for 20 min and then to $456 \mathrm{mM}$ ethanol for $30 \mathrm{~min}$. The third group served as an untreated control. The mid brain of each fish was kept in an aluminium sample pan and its free water level was assessed by differential scanning calorimetry (DSC). Data were analyzed by ANOVA.

Results and discussion: The results show that there was no significant variation in melting and freezing temperature of brain samples but the enthalpies, both freezing and melting varied significantly $(\mathrm{P}<0.05)$ between Nux vom treated group and the untreated groups. All alcohol treated fish showed significant reduction in the level of free water molecules as compared to the untreated control. Treatment with Nux vomica increased the level of free water in the brain significantly as compared to the untreated alcoholic group.
\end{abstract}

Conclusion: Alcohol intoxication reduces free water molecules in the fish brain. Nux vom might have acted on aquaporins in the glial cells thereby increasing the level of free water in the brain.

Keywords: Free water molecules, Aquaporin 4, Nux vomica, Alcohol, Brain.

Received: March 1, 2018. Accepted: April 26, 2018.

(C) International Journal of High Dilution Research.

Not for commercial purposes. 